Review

\title{
Exosomes in Coronary Artery Disease
}

\author{
Xiao-Fei Gao1,2, Zhi-Mei Wang11, Feng Wang1, Yue Gu11, Jun-Jie Zhang ${ }^{1,2}{ }^{\circledR}$, Shao-Liang Chen ${ }^{1,2}$ \\ 1. Department of Cardiology, Nanjing First Hospital, Nanjing Medical University, Nanjing, China; \\ 2. Department of Cardiology, Nanjing Heart Centre, Nanjing, China. \\ The first two authors contributed equally to this work
}

$\triangle$ Corresponding authors: Dr. Jun-Jie Zhang, MD, PhD. Department of Cardiology, Nanjing First Hospital, Nanjing Medical University. No. 68 Changle Road, 210006 Nanjing, China; Telephone \& Fax: +86-25-52208048; E-mail:jameszll@163.com; and Dr. Shao-Liang Chen, MD, PhD. Department of Cardiology, Nanjing First Hospital, Nanjing Medical University. No. 68 Changle road, 210006 Nanjing, China; Telephone \& Fax: +86-25-52208048; E-mail: chmengx@126.com

(1) The author(s). This is an open access article distributed under the terms of the Creative Commons Attribution License (https://creativecommons.org/licenses/by/4.0/). See http://ivyspring.com/terms for full terms and conditions.

Received: 2019.05.06; Accepted: 2019.08.01; Published: 2019.09.07

\begin{abstract}
Exosomes, the nanosized vesicles released from various cell types, contain many bioactive molecules, such as proteins, lipids, and nucleic acids, which can participate in intercellular communication in a paracrine manner or an endocrine manner, in order to maintain the homeostasis and respond to stress adaptively. Currently, exosomes have already been utilized as diagnostic biomarkers and therapeutic tools in cancer clinical trials. There has also been great progress in cell and animal exosomes studies of coronary artery disease (CAD). Emerging evidence suggests that exosomes released from endothelial cells, smooth muscle cells, adipose cells, platelets, cardiomyocytes, and stem cells have been reported to play crucial roles in the development and progression of CAD. Moreover, it has been showed that exosomes released from different cell types exhibit diverse biological functions, either detrimental or protective, depending on the cell state and the microenvironment. However, the systematic knowledge of exosomes in CAD at the patient level has not been well established, which are far away from clinical application. This review summarizes the basic information about exosomes and provides an update of the recent findings on exosome-mediated intercellular communication in the development and progression of CAD, which could be helpful for understanding the pathophysiology of CAD and promoting the further potential clinical translation.
\end{abstract}

Key words: extracellular vesicles, exosomes, coronary artery disease

\section{Introduction}

Coronary artery disease (CAD) is a common condition that strongly correlates with increased cardiovascular morbidity and mortality, and it poses a high economic burden to the national healthcare system [1,2]. An overall better understanding of the mechanisms of atherosclerosis, the development of antiplatelet and statin therapies, and advances in devices and procedural techniques for coronary intervention have contributed to a great improvement in the clinical prognosis of patients [3]. Despite this, patients with coronary disease still face the residual risks of stent restenosis, cardiac remodeling, and ischemia/reperfusion injury. Thus, further exploration of the pathophysiology of CAD is important, and a novel, reliable tool for these patients will be of great interest.

Extracellular vesicles, membrane-bound organelles and released by diverse cell types, contain many bioactive molecules, such as proteins, lipids, and nucleic acids; these vesicles are varied in size, biogenesis and contents, which usually include apoptotic bodies, microvesicles (also called ectosomes, microparticles, or matrix vesicles), and exosomes [4,5]. The similarities and differences of these three vesicles are summarized in Table 1. In brief, apoptotic bodies are large vesicles (more than 1 $\mu \mathrm{m}$ in diameter) with a permeable membrane that are formed upon cell apoptosis [5,6]. Microvesicles (0.1-1 $\mu \mathrm{m}$ in diameter) are generated by shedding directly from the plasma membrane and undergo the process 
of cytoskeleton remodeling and the externalization of phosphatidylserine [5,7]. Exosomes are the smallest vesicles (30-150 nm in diameter) of endosomal origin and are released into extracellular space upon the fusion of multivesicular bodies (MVBs) with the plasma membrane, which occurs in an endosomal sorting complex required for transport (ESCRT)dependent or ESCRT-independent manner [7-9].

In the past decade, emerging evidence in the field of cardiovascular disease [10-16] reported that exosomes play crucial roles in intercellular communication via transferring cargo (including proteins, RNA, DNA, and other molecules) under not only physiological situations (angiogenesis and cardiac growth) but also pathological conditions (ischemia/reperfusion injury, vascular calcification, atherosclerosis, and cardiac remodeling). In this review, we focus on the updated findings in exosomes biogenesis, secretion and uptake, isolation and detection, as well as exosomes-mediated intercellular communication in the development and progression of CAD.

\section{Exosomes Biogenesis, Secretion and Uptake}

The explicit mechanisms of exosomes biogenesis are quite complicated and only partly understood $[17,18]$. In brief, early endosomes, which are formed by the inward budding of the cell membrane, can mature into late endosomes [19,20]. Intraluminal vesicles (ILVs), also called exosomes precursors, originate in the lumen of late endosomes by the inward budding of the endosomal membrane [21]. The late endosomes that contain ILVs become MVBs, which have two possible outcomes [22]: either fusing with the plasma membrane to release the ILVs to the extracellular space (called exosomes) or fusing with lysosomes for lysosomal degradation (Figure 1).
Several key molecules have been verified to participate in membrane fusion and the release of exosomes into the extracellular space. Small Rab GTPases were identified to promote exosomes secretion, and Rab27a and Rab27b were involved in MVB docking at the plasma membrane [23]. Hyenne et al [24] also found that RAL-1 (Ras-related GTPase homolog) controlled MVB formation and exosomes secretion. Moreover, soluble N-ethylmaleimidesensitive factor attachment protein receptors (SNAREs) are important molecules for vesicle fusion [6,25-27]. Recently, Zou et al [28] found that exosomes release, such as autophagy, could be regulated by the mechanistic target of rapamycin complex 1 (mTORC1) in response to changes in amino acids and growth factor conditions, both in cultured cells and in vivo.

After secretion, exosomes in the extracellular space can be taken up by recipient cells to affect the gene expression and function of recipient cells. There are three known types of exosomes uptake mechanisms as follows: fusion, binding, and endocytosis (Figure 1) [29,30]. After exosomal membranes directly fuse with recipient cell membranes, the cargo, including proteins, miRNAs, mRNAs, etc. are released into the cytoplasm of recipient cells. Exosomes, with external ligands [31], can also bind to the receptors of recipient cells to activate signal transduction. Moreover, the mechanisms of endocytosis $[9,30,32,33]$ (the most frequent type) vary greatly and include macropinocytosis, phagocytosis, caveolae-mediated endocytosis, lipid raft-mediated endocytosis, and clathrin-mediated endocytosis; the type of endocytosis may depend on the cell types and physiological conditions. For example, exosomes uptake may occur via extracellular signal-regulated kinase 1/2 (ERK 1/2) and heat shock protein 27 (HSP27) signaling or lipid raft-mediated endocytosis, which was negatively regulated by caveolin-1 during exosomes internalization [34].

Table 1. Characterization and Classification of Extracellular Vesicles.

\begin{tabular}{|c|c|c|c|}
\hline Characterization & Exosomes & Microvesicles & Apoptotic bodies \\
\hline Similarities & \multicolumn{3}{|c|}{$\begin{array}{l}\text { Present in all biofluids } \\
\text { Released from almost all types of cells } \\
\text { Includes proteins, lipids, and nucleic acids } \\
\text { Isolation: (ultra) centrifugation } \\
\text { Identification: electron microscope for morphology; NTA for size and concentration; western blot for biomarker expression } \\
\text { Intercellular communication: transferring biologic information to recipient cells }\end{array}$} \\
\hline Size (diameter) & $\sim 30-150 \mathrm{~nm}$ & $\sim 0.1-1 \mu \mathrm{m}$ & $1-5 \mu \mathrm{m}$ \\
\hline Formation & Fusion of MVB with cell membrane & Outward budding of the plasma membrane & Release after cell apoptosis \\
\hline Formation pathways & $\begin{array}{l}\text { ESCRT pathways; Tetraspanin or ceramide } \\
\text { pathways }\end{array}$ & $\begin{array}{l}\text { Various enzymes and mitochondrial or calcium signaling pathways } \\
\text { (complex and unclear) }\end{array}$ & Apoptosis-related pathway \\
\hline Markers & $\begin{array}{l}\text { CD9, CD63, CD81 } \\
\text { TSG 101, flotillin, annexin } \\
\text { HSP70, HSP90 }\end{array}$ & $\begin{array}{l}\text { Integrins, selections, and other antigens from parent cells } \\
\text { High phosphatidylserine exposure }\end{array}$ & $\begin{array}{l}\text { High phosphatidylserine } \\
\text { exposure } \\
\text { Caspase } 3 \text {, histones }\end{array}$ \\
\hline
\end{tabular}

NTA: nanoparticle tracking analysis; MVB: multivesicular bodies; ESCRT: endosomal sorting complex required for transport; CD: cluster differentiation; TSG: tumor susceptibility gene. 


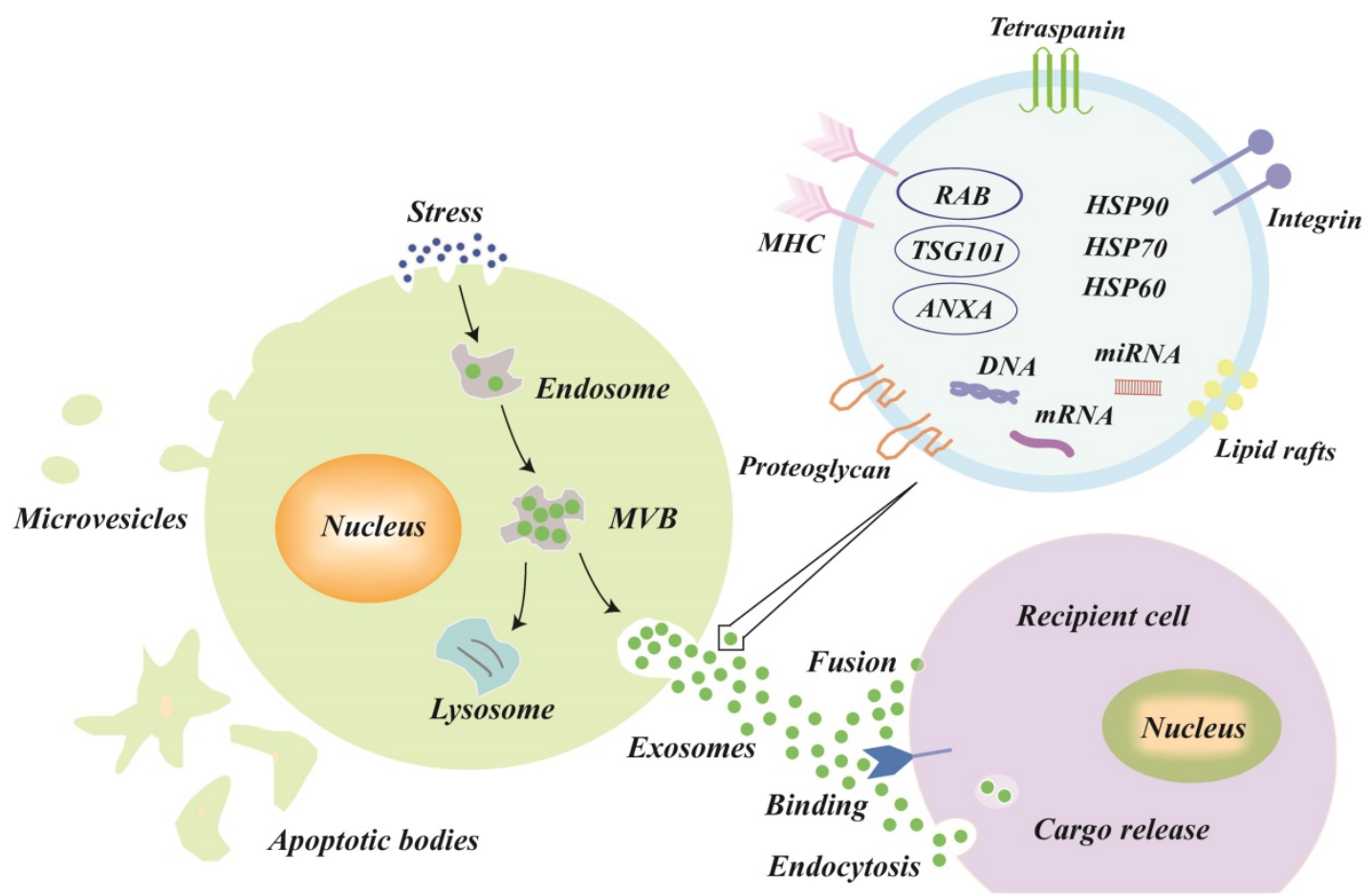

Figure 1. Biogenesis, secretion, uptake and the schematic presentation of exosomes. Endosomes are formed by the inward budding of the cell membrane. Multivesicular bodies (MVBs) originate in the lumen of the endosomes by an inward budding of the endosomal membrane, which has two outcomes: either fusing with the plasma membrane to release its intraluminal vesicles to the extracellular space (called exosomes) or fusing with lysosomes for lysosomal degradation. The three types of mechanisms of exosome uptake by recipient cells are fusion, binding, and endocytosis. In general, the exosomes are formed with the following molecules: tetraspanin family (CD9, CD63, and CD81), lipid rafts, integrin, tumor susceptibility gene 101 (TSG101), major histocompatibility complex (MHC) class II molecules, heat shock proteins (HSP60, HSP70, and HSP90), annexins, and nucleic acids.

\section{Exosomes Isolation and Detection}

The current known contents of exosomes include at least 41,860 proteins, more than 7,540 RNAs and 1,116 lipid molecules [35], and the content of the exosomes varies greatly according to their parent cell type and microenvironment [36-40]. In general, the exosomes are formed with the following molecules: tetraspanin family (CD9, CD63, and CD81 (transmembrane proteins)), tumor susceptibility gene 101 (TSG101, used for sorting and transporting exosomes), major histocompatibility complex (MHC) class II molecules, and programmed cell death 6-interacting proteins (PDCD6IPs) [18,22,37,38,41]. Additionally, heat shock proteins (HSP60, HSP70, and HSP90), cytoskeletal proteins (actin and tubulin), annexins (regulate cytoskeletal changes in membranes and membrane fusion), and membrane transport proteins are also present in exosomes (Figure 1), irrespective of the type [18,22,38,41].

Exosomes could be isolated from body fluids or cell culture media by several different techniques, including differential and gradient density centrifugation, filtration, immunological separation, precipitation, and commercial exosomes isolation kits $[18,41,42]$. Briefly, centrifugation-based techniques are the most common method to isolate exosomes, and this method is also regarded as the gold standard for exosomes isolation [43], but this method has the following limitations: a large sample volume, time-consuming, and a low exosomes recovery (5\%-25\%) [44-46]. Filtration-based techniques are currently used to eliminate dead cells and debris, which prepares the sample for further ultracentrifugation $[18,47]$. Immunological separation has a high specificity because antibody-coated magnetic beads bind to the proteins in the exosomes, but this technique was blamed for a high cost and a low exosome yield $[46,48]$. The exosome precipitation method is utilized in several commercial exosomes isolation kits and has many advantages, including simplicity, low sample volume, and cost-effectiveness $[47,49]$. However, the precipitated samples are mixed with contaminants (proteins, lipids, etc.), which are not suitable for direct further detection accurately. Of note, a recent study [50] found that different cell culture conditions and isolation methods could result in varying glycosylated exosomes populations. Overall, the selection of isolation method should be based on the down-stream application and the experiment purpose.

The morphology of exosomes could be detected by a variety of electron microscopy techniques, including transmission electron microscopy (TEM), scanning electron microscopy, cryo-electron microscopy and atomic force microscopy [18]. 
Western blots and (high resolution) flow cytometry are widely used to identify the specific surface protein markers of exosomes. The size of the exosome is measured by nanoparticle tracking analysis (NTA), tunable resistive pulse sensing, and dynamic light scattering [22,51]. In the current basic research on exosomes, combinations of TEM, western blots (or flow cytometry), and NTA are the most widely used tools to detect exosomes. Moreover, according to minimal experimental requirements for the definition of extracellular vesicles from the International Society for Extracellular Vesicles [22,41], exosomes are defined as vesicles that contain at least one transmembrane protein (CD9, CD63, CD81, adhesion molecules, etc.) and one cytosolic protein (TSG101, annexins, Rabs, etc.), without any endoplasmic reticulum proteins (calnexin and Golgi matrix proteins) or nuclear proteins.

Several key points are quite crucial for the exosome isolation and detection. To exclude the effects of exosomes in fetal bovine serum (FBS), more than $100,000 \times \mathrm{g}$ ultracentrifugation of complete medium (or of FBS following at least 1:4 dilution) for at least 18 hours should be performed [41].
Commercial exosomes-depleted FBS is also available from vendors. Additionally, the storage temperature of exosomes is very important. Exosomes storage at $-80^{\circ} \mathrm{C}$ for three to six months could maintain the basic functions, but current view suggests that original samples are stored at $-80^{\circ} \mathrm{C}$ and exosomes are isolated just before the index experiments [41,52-54].

\section{Exosomes and Coronary Artery Disease}

The specific mechanism of atherosclerosis is complex, and the mechanism is a chronic process with multifactorial causes. The current view [55-58] is that oxidative stress, endothelial dysfunction, and inflammation are three key factors involved in the development and progression of atherosclerosis. Exosomes participate in cardiovascular cell-to-cell (paracrine) and distant (endocrine) communication via miRNAs and other mediators [59]. Exosomes released from stem cells, endothelial cells, smooth muscle cells, cardiomyocytes, adipose cells, and platelets include potential valuable biological information for the development and progression of CAD (Figure 2) [5].

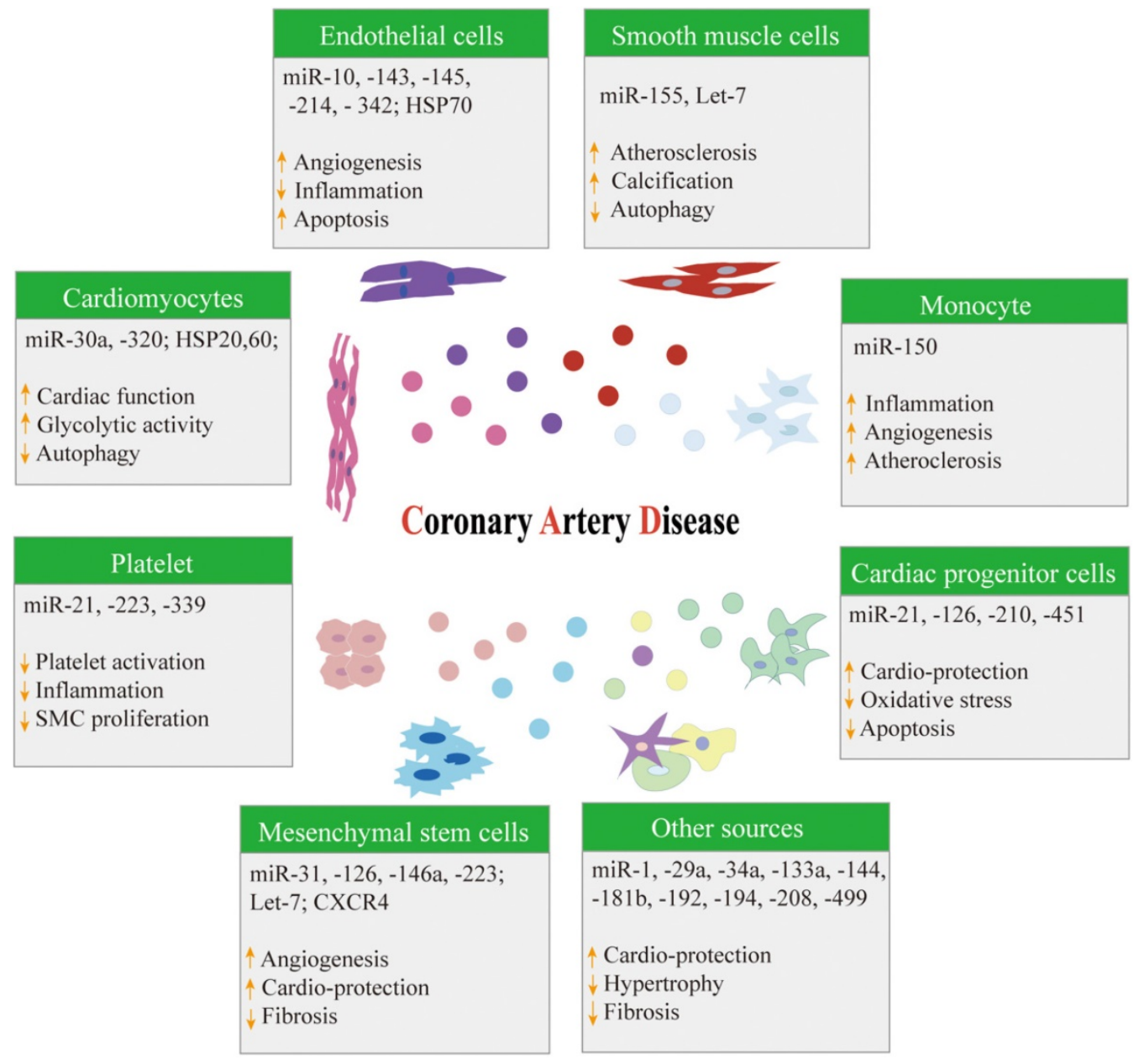

Figure 2. A summary of different cell-derived exosomes in coronary artery disease. HSP: Heat shock protein; SMC: Smooth muscle cell; CXCR4: C-X-C chemokine receptor type 4. 


\section{Cardiac progenitor cell-derived exosomes}

Exosomes released by cardiac progenitors have been shown to prevent ischemic myocardium against acute ischemia/reperfusion injury through miR-451 [60]. Cardiac progenitor cell-derived exosomes could also protect cardiomyocytes against oxidative stress-related apoptosis via exosomal miR-21 by targeting programmed cell death 4 (PDCD4) [61]. Moreover, the exosomal transfer of miR-126 and miR-210 from host cells to transplanted cells could improve the survival of transplanted cardiac progenitor cells into the ischemic myocardium [62].

\section{Mesenchymal stem cell-derived exosomes}

Exosomes derived from mesenchymal stem cells (MSCs) improved atherosclerosis in ApoE-/-mice and promoted M2 macrophage polarization in the atherosclerotic plaque by the microRNA-let7/ HMGA2/NF-kB pathway [63]. Kang et al [64] also reported that $\mathrm{C}-\mathrm{X}-\mathrm{C}$ chemokine receptor type 4 (CXCR4)-overexpressing MSC-derived exosomes could reduce infarct size, improve cardiac remodeling, and increase angiogenesis by activating the Akt signaling pathway following myocardial infarction. Furthermore, exosomes released from MSCs regulated the protein expression of adherent junctions, leading to angiogenesis via miRNA 31 [65], miRNA 126 [65], miRNA 146a [66], and miRNA 223 [67].

\section{Endothelial cell-derived exosomes}

Endothelial cell-derived exosomes can reduce ischemia and reperfusion injury in cardiomyocytes via the activation of the ERK1/2 Mitogen-activated protein kinase (MAPK) signaling pathway and may contribute to ischemic preconditioning [68]. Zhan et al [69] demonstrated that oxidative low density lipoprotein (OX-LDL) and homocysteine promoted the activation of endothelial cells, the secretion of exosomes, and the increased HSP70 content of exosomes from endothelial cells; HSP70, which further activated monocytes, lead to monocyte adhesion to endothelial cells. Exosomal metastasis-associated lung adenocarcinoma transcript 1 (MALAT1) was derived from OX-LDL-treated endothelial cells and promoted M2 macrophage polarization, which is defined as an increase in the expression of M2 macrophage markers (CD206, arginase-1, and IL-10) and decreases in the expression of an M1 macrophage marker (IL-12) [70]. Hergenreider et al [71] also found that exosomes released by Krüppel-like factor (KLF) 2-transduced endothelial cells transferred to smooth muscle cells and reduced atherosclerotic lesion formation in a miRNA 143/145-dependent manner. Moreover, endothelial cell-derived miR-214-containing exosomes could inhibit senescence and promote angiogenesis in human and mouse endothelial cells [72]. Njock et al [73] reported that exosomal miR-10a released from endothelial cells was taken up by monocytic cells and could inhibit inflammatory signaling via targeting the NF-kB pathway. Recently, Hou et al [74] found that laminar shear stress or exercise training directly increased miR-342-5p synthesis in endothelial cells and increased the miR-342-5p-enriched exosomes that inhibited cardiomyocyte apoptosis after myocardial ischemia/reperfusion injury by targeting Caspase 9 and JNK2; in addition, the stress improved survival signaling ( $\mathrm{p}-\mathrm{Akt}$ ) by targeting the phosphatase gene Ppm1f.

\section{Smooth muscle cell-derived exosomes}

Vascular smooth muscle cell-derived exosomes could mediate the transfer of KLF 5-induced miR-155 from smooth muscle cells to endothelial cells, which increases endothelial permeability and promotes atherosclerotic plaque progression [75]. Ding et al [76] reported that vascular smooth muscle cells that were treated with hsa-let-7g could inhibit autophagy and apoptosis, also leading to reduced intracellular ROS generation. Moreover, exosomes have been demonstrated to play crucial roles in vascular calcification by initiating mineral deposition [77,78]; exosomes also can regulate the process of vascular calcification via transporting microRNAs to recipient smooth muscle cells [78-80]. An in vivo study showed that exosomes were found in arteries from patients with chronic kidney disease, and CD63 (a biomarker of exosomes) was observed to colocalize with calcification [81]. Cytokines and growth factors were also found to promote exosome release, contributing to smooth muscle cell calcification in response to environmental calcium stress [81,82].

\section{Cardiomyocyte-derived exosomes}

Gupta et al [33] first reported the exosomes released by cardiomyocytes, and exosomes containing HSP60 could protect cells against myocardial infarction. The elevation of HSP20 in cardiomyocytes also improved cardiac function and angiogenesis via activating exosomes biogenesis in diabetic mice [83]. Moreover, cardiomyocyte-derived exosomes could regulate glycolytic flux in endothelial cells by the direct transfer of glycolytic enzymes and GLUT transporters [84]. Exosomes released from hypoxic cardiomyocytes also inhibited autophagy by transferring miRNA-30a between cardiomyocytes in a paracrine manner [85]. Notably, Wang et al [86] found that exosomes released by cardiomyocytes could exert 
an anti-angiogenic function (the inhibition of endothelial cell proliferation, migration and tube formation) in type 2 diabetic rats through the exosomal transfer of miR-320 into endothelial cells. In addition, cardiomyocyte-derived exosomes in infarcted hearts have been demonstrated to increase the injury of transplanted bone marrow mesenchymal stem cells [87]. Hypoxia inducible factor-1a (HIF-1a) also initiated TNF-a expression in acute myocardial infarction, which was mediated by exosomes derived from cardiomyocytes [88].

\section{Dendritic cells and monocyte-derived exosomes}

Gao et al [89] showed that exosomes derived from mature dendritic cells are involved in endothelial inflammation through the membrane TNF-a-mediated NF-kB pathway and that exosomes could be taken up by aortic endothelial cells and could induce inflammation and atherosclerosis. Exosomes released from primary human monocytes, dendritic cell precursors, could be taken up by endothelial cells; this contributes to endothelial dysfunction via the TLR4 and NF-kB pathways [90]. The monocyte-derived exosomes mediated transfer of microRNA-150 from monocytes to endothelial cells can enhance the capillary tube formation of endothelial cells and promote angiogenesis [91]. Moreover, another study [92] reported that exosomes secreted from LPS-activated macrophages affected the gene expression and differentiation of adipocyte, which might play a critical role in atherosclerosis. Chronic inflammation is a crucial factor involved in atherosclerosis, and dendritic cell and monocyte-derived exosomes promote the development and progression of coronary artery disease.

\section{Adipose cell-derived exosomes}

Clinical study [93] showed that the imbalance of epicardial adipose tissue volume and adipocytokine was strongly related to coronary atherosclerosis. Adipose cell-derived circulating exosomes miRNAs could regulate gene expression in distant tissues [94]. Adipose cell-derived exosomes also have been reported to mediate the activation of TNF- $\alpha$ and IL-6 in macrophages and insulin resistance via the TLR4/ Toll-interleukin-1 receptor domain-containing adaptor protein inducing interferon- $\beta$ (TRIF) pathway [95]. Exosomes derived from insulin resistance adipocyte in diabetic ApoE deficit mice could promote atherosclerosis and vulnerable plaque via vasa vasorum angiogenesis [96]. Additionally, a recent study showed that cardiomyocytes, uptake adipose cell-derived exosomal miR-214 through clathrin-mediated endocytosis, could prevent cardiomyocyte damage after acute myocardial infarction [97]. Exosomes released by adipose cell-derived MSC have been demonstrated to decrease cell adhesion molecules expression via inhibiting the MAPK and NFkB pathways and reduce atherosclerosis in LDL receptor-deficient mice [98].

\section{Platelet-derived exosomes}

Fifteen years ago, Caby et al [99] demonstrated that blood was a physiological fluid for exosomes circulation, indicating an important role of exosomes in cell-to-cell communication through the transfer of cellular material to reach distant recipient cells. In $\mathrm{FeCl}_{3}$-induced murine carotid arteries, platelet-derived exosomes could suppress atherothrombotic processes by enhancing the protein ubiquitination and proteasome degradation of CD36 (a type II scavenger receptor in platelets) and by inhibiting platelet activation and thrombosis [100]. Thrombin-activated platelet-derived exosomes inhibit the endothelial cell expression of intercellular adhesion molecule-1 (ICAM-1) by microRNA-223 during inflammation [101]. Thrombin-stimulated platelet-derived exosomes containing miR-223, miR-339 and miR-21 inhibit platelet-derived growth factor receptor- $\beta$ expression in vascular smooth muscle cells [102]. Overall, platelet-derived exosomes may serve as therapeutic targets of coronary artery disease by inhibiting platelet activation, suppressing inflammation, and reducing the proliferation of smooth muscle cells.

\section{Exosomes from other sources}

Plasma exosomes from rats and healthy volunteers exhibit cardioprotective functions against ischemia-reperfusion injury through the HSP70/TLR4 communication axis [103]. Exosomes secretion after ischemic preconditioning is involved in cardioprotection by remote ischemic preconditioning, underlining the importance of exosomal transfer mechanisms in remote cardioprotection [104]. Exosomal miR-29a, a key regulator of tissue fibrosis, leads to the beneficial effect of remote ischemic conditioning to improve left ventricular remodeling on chronic heart failure after acute myocardial infarction [105]. Exosomal miR-144 could play a crucial role in the cardioprotection induced by remote ischemic preconditioning, and miR-144 might serve as a novel therapeutic target to decrease clinical ischemia-reperfusion injury [106]. Exosomal miR-1 and miR-133a were derived from the injured myocardium [107] after acute myocardial infarction and could be transferred to adjacent myocardium to protect cardiomyocytes against hypertrophy. 
Moreover, Cheng et al [108] reported that urine exosomal miR-1 could be a novel urine biomarker for acute myocardial infarction. Matsumoto et al [109] demonstrated that circulating microRNAs (miR-34a, miR-192, and miR-194) were predictive indicators of ischemic heart failure via the p53 pathway in patients with acute myocardial infarction. The exosomes released from the human cardiac muscle patches (human induced-pluripotent stem cells) were shown to have cardioprotective effects that increased the survival of cardiomyocytes [110]. A porcine model [111] showed that cardiosphere-derived exosomes could decrease scarring after myocardial infarction, reduce adverse remodeling and increase left ventricular ejection fraction in acute and convalescent myocardial infarctions. Exosomal miR-181b from cardiosphere-derived cells could induce macrophage polarization and reduce protein kinase $C$ transcript levels; in addition, they exhibit a cardioprotective effect in acute myocardial infarction [112]. Recently, Cheng et al [113] found that circulating exosomes carrying myocardial-miRs (miR-1, miR-208, and miR-499) were transferred selectively to peripheral organs, preferentially to the bone marrow, and that these transferred myocardial-miRs downregulated CXCR4 expression in bone marrow cells, leading to progenitor cell mobilization.

\section{Exosomes and potential diagnostic implication in CAD}

Limited studies reported the potential diagnostic implication of exosomes in CAD [12]. A previous clinical study [114] showed that serum exosomes of patients with acute coronary disease (ACS) presented with a high expression of miR-208a compared with that in healthy subjects. More importantly, patients with low miR-208a expression were associated with a significant lower mortality rate than those with high miR-208a expression $(3.3 \%$ vs. $11.0 \%, \mathrm{p}<0.05)$ during one-year follow-up. Another study [115] found that the plasma exosomes and exosomal cardiac microRNAs could be increased significantly in patients undergoing coronary artery bypass surgery for up to $48 \mathrm{~h}$ after surgery, meanwhile these exosomes and their microRNAs could be positively related to high sensitive cardiac troponin (cardiac biomarker).

\section{Conclusions and perspectives}

In this review, we have discussed exosomes biogenesis, secretion, uptake, isolation and detection, and we have discussed exosomes-mediated intercellular communication in CAD. Exosomes, such as those carrying proteins, lipids, and nucleic acids, could be taken up by the recipient cells in a paracrine or endocrine manner. Exosomes released from different cell types exhibit various biological functions, either detrimental or protective, depending on the cell state and the microenvironment.

Exosomes isolation, detection and purification should be standardized and simplified to ensure that exosomes analysis and application are feasible in daily clinical practice. The underlying mechanisms of exosomes biogenesis, uptake and clearance also need to be explored deeply; then, exosomes may serve as a clinical delivery tool of novel drugs for coronary artery disease. Moreover, a detailed characterization and functional assessment of exosomes is essential to confirm the full therapeutic effect on coronary artery disease. Therefore, the clinical translation of exosomes in the diagnosis and treatment of coronary artery disease is full of hope, but it still has a long way to go.

\section{Abbreviations}

$\mathrm{CD}$, cluster differentiation; CAD, coronary artery disease; CXCR4, C-X-C chemokine receptor type 4; ERK, extracellular signal-regulated kinase; ESCRT, endosomal sorting complex required for transport; HIF, hypoxia-inducible factor; HSP, heat shock protein; ICAM-1, intercellular adhesion molecule-1; ILVs, intraluminal vesicles; KLF, Krüppel-like factor; MHC, major histocompatibility complex; MSCs, mesenchymal stem cells; mTORC, mechanistic target of rapamycin complex; MAPK, mitogen-activated protein kinase; MVB, multivesicular bodies; NTA, nanoparticle tracking analysis; OX-LDL, oxidative low density lipoprotein; SMC, smooth muscle cell; SNAREs, soluble N-ethylmaleimide-sensitive factor attachment protein receptors; TEM, transmission electron microscopy; TRIF: Toll-interleukin-1 receptor domain-containing adaptor protein inducing interferon- $\beta$; TSG: tumor susceptibility gene.

\section{Acknowledgments}

Contributions of authors: Xiao-Fei Gao and Zhi-Mei Wang contributed to literature search and drafted the manuscript; Feng Wang and Yue Gu contributed to literature search; Jun-Jie Zhang and Shao-Liang Chen contributed to review design, wrote and revised the manuscript.

Funding sources: This study was granted by the National Natural Science Foundation of China (NSFC 81270191, NSFC 91439118, and NSFC 91639303), and was jointly supported by Six Talent Peaks Project in Jiangsu Province (2014-WSN-058), Nanjing Health and Family Planning Commission (YKK16124), Nanjing Health Youth Talent Training project (QRX17017), and Nanjing Municipal Commission of Science \& Technology (201715026). 


\section{Competing Interests}

The authors have declared that no competing interest exists.

\section{References}

1. Hao Y, Liu J, Liu J, Yang N, Smith SC, Jr., Huo Y, et al. Sex Differences in In-hospital Management and Outcomes of Patients with Acute Coronary Syndrome: Findings from the Improving Care for Cardiovascular Disease in China (CCC) Project. Circulation. 2019.

2. Benjamin EJ, Muntner P, Alonso A, Bittencourt MS, Callaway CW, Carson AP, et al. Heart Disease and Stroke Statistics-2019 Update: A Report From the American Heart Association. Circulation. 2019; 139(10):e56-e66.

3. Neumann FJ, Sousa-Uva M, Ahlsson A, Alfonso F, Banning AP, Benedetto U, et al. 2018 ESC/EACTS Guidelines on myocardial revascularization. Eur Heart J. 2018

4. Colombo M, Raposo G, Thery C. Biogenesis, secretion, and intercellular interactions of exosomes and other extracellular vesicles. Annu Rev Cell Dev Biol. 2014; 30:255-289.

5. Boulanger CM, Loyer X, Rautou PE, Amabile N. Extracellular vesicles in coronary artery disease. Nat Rev Cardiol. 2017; 14(5):259-272.

6. Pfeffer SR. Unsolved mysteries in membrane traffic. Annu Rev Biochem. 2007; 76:629-645.

7. Meldolesi J. Exosomes and Ectosomes in Intercellular Communication. Curr Biol. 2018; 28(8):R435-R444

8. Stoorvogel W. Resolving sorting mechanisms into exosomes. Cell Res. 2015; 25(5):531-532.

9. van Niel G, D'Angelo G, Raposo G. Shedding light on the cell biology of extracellular vesicles. Nat Rev Mol Cell Biol. 2018; 19(4):213-228.

10. Poe AJ, Knowlton AA. Exosomes as agents of change in the cardiovascular system. J Mol Cell Cardiol. 2017; 111:40-50.

11. Shanmuganathan M, Vughs J, Noseda M, Emanueli C. Exosomes: Basic Biology and Technological Advancements Suggesting Their Potential as Ischemic Heart Disease Therapeutics. Front Physiol. 2018; 9:1159.

12. Jansen F, Nickenig G, Werner N. Extracellular Vesicles in Cardiovascular Disease: Potential Applications in Diagnosis, Prognosis, and Epidemiology. Circ Res. 2017; 120(10):1649-1657.

13. Bellin G, Gardin C, Ferroni L, Chachques JC, Rogante M, Mitrecic D, et al. Exosome in Cardiovascular Diseases: A Complex World Full of Hope. Cells. 2019; 8(2).

14. Shah R, Patel T, Freedman JE. Circulating Extracellular Vesicles in Human Disease. N Engl J Med. 2018; 379(10):958-966

15. Hafiane A, Daskalopoulou SS. Extracellular vesicles characteristics and emerging roles in atherosclerotic cardiovascular disease. Metabolism. 2018; 85:213-222.

16. Osteikoetxea X, Nemeth A, Sodar BW, Vukman KV, Buzas EI. Extracellular vesicles in cardiovascular disease: are they Jedi or Sith? J Physiol. 2016; 594(11):2881-2894

17. Pegtel DM, Gould SJ. Exosomes. Annu Rev Biochem. 2019; 88:487-514

18. Boriachek K, Islam MN, Moller A, Salomon C, Nguyen NT, Hossain MSA, et al. Biological Functions and Current Advances in Isolation and Detection Strategies for Exosome Nanovesicles. Small. 2018; 14(6)

19. Thery C, Zitvogel L, Amigorena S. Exosomes: composition, biogenesis and function. Nat Rev Immunol. 2002; 2(8):569-579.

20. Stoorvogel W, Strous GJ, Geuze HJ, Oorschot V, Schwartz AL. Late endosomes derive from early endosomes by maturation. Cell. 1991; 65(3):417-427.

21. Huotari J, Helenius A. Endosome maturation. EMBO J. 2011; 30(17):3481-3500.

22. Lotvall J, Hill AF, Hochberg F, Buzas EI, Di Vizio D, Gardiner C, et al. Minimal experimental requirements for definition of extracellular vesicles and their functions: a position statement from the International Society for Extracellular Vesicles. J Extracell Vesicles. 2014; 3:26913.

23. Ostrowski M, Carmo NB, Krumeich S, Fanget I, Raposo G, Savina A, et al. Rab27a and Rab27b control different steps of the exosome secretion pathway. Nat Cell Biol. 2010; 12(1):19-30; sup pp 11-13.

24. Hyenne V, Apaydin A, Rodriguez D, Spiegelhalter C, Hoff-Yoessle S, Diem M, et al. RAL-1 controls multivesicular body biogenesis and exosome secretion. J Cell Biol. 2015; 211(1):27-37.

25. Hessvik NP, Llorente A. Current knowledge on exosome biogenesis and release. Cell Mol Life Sci. 2018; 75(2):193-208.

26. Wei Y, Wang D, Jin F, Bian Z, Li L, Liang H, et al. Pyruvate kinase type M2 promotes tumour cell exosome release via phosphorylating synaptosome-associated protein 23. Nat Commun. 2017; 8:14041.

27. Fader CM, Sanchez DG, Mestre MB, Colombo MI. TI-VAMP/VAMP7 and VAMP3/cellubrevin: two v-SNARE proteins involved in specific steps of the autophagy/multivesicular body pathways. Biochim Biophys Acta. 2009; 1793(12):1901-1916.

28. Zou W, Lai M, Zhang Y, Zheng L, Xing Z, Li T, et al. Exosome Release Is Regulated by mTORC1. Adv Sci (Weinh). 2019; 6(3):1801313.

29. Morrison EE, Bailey MA, Dear JW. Renal extracellular vesicles: from physiology to clinical application. J Physiol. 2016; 594(20):5735-5748.

30. Mulcahy LA, Pink RC, Carter DR. Routes and mechanisms of extracellular vesicle uptake. J Extracell Vesicles. 2014; 3
31. Patel B, Patel J, Cho JH, Manne S, Bonala S, Henske E, et al. Exosomes mediate the acquisition of the disease phenotypes by cells with normal genome in tuberous sclerosis complex. Oncogene. 2016; 35(23):3027-3036.

32. Nanbo A, Kawanishi E, Yoshida R, Yoshiyama H. Exosomes derived from Epstein-Barr virus-infected cells are internalized via caveola-dependent endocytosis and promote phenotypic modulation in target cells. J Virol. 2013; 87(18):10334-10347.

33. Gupta S, Knowlton AA. HSP60 trafficking in adult cardiac myocytes: role of the exosomal pathway. Am J Physiol Heart Circ Physiol. 2007; 292(6):H3052-3056

34. Svensson KJ, Christianson HC, Wittrup A, Bourseau-Guilmain E, Lindqvist E, Svensson LM, et al. Exosome uptake depends on ERK1/2-heat shock protein 27 signaling and lipid Raft-mediated endocytosis negatively regulated by caveolin-1. J Biol Chem. 2013; 288(24):17713-17724.

35. Keerthikumar S, Chisanga D, Ariyaratne D, Al Saffar H, Anand S, Zhao K, et al. ExoCarta: A Web-Based Compendium of Exosomal Cargo. J Mol Biol. 2016; 428(4):688-692.

36. Hosseini-Beheshti E, Pham $\mathrm{S}$, Adomat $\mathrm{H}, \mathrm{Li}$ N, Tomlinson Guns ES. Exosomes as biomarker enriched microvesicles: characterization of exosomal proteins derived from a panel of prostate cell lines with distinct AR phenotypes. Mol Cell Proteomics. 2012; 11(10):863-885.

37. Simpson RJ, Jensen SS, Lim JW. Proteomic profiling of exosomes: current perspectives. Proteomics. 2008; 8(19):4083-4099.

38. Zaborowski MP, Balaj L, Breakefield XO, Lai CP. Extracellular Vesicles: Composition, Biological Relevance, and Methods of Study. Bioscience. 2015; 65(8):783-797.

39. Huang X, Yuan T, Liang M, Du M, Xia S, Dittmar R, et al. Exosomal miR-1290 and miR-375 as prognostic markers in castration-resistant prostate cancer. Eur Urol. 2015; 67(1):33-41.

40. Skotland T, Sandvig K, Llorente A. Lipids in exosomes: Current knowledge and the way forward. Prog Lipid Res. 2017; 66:30-41.

41. Thery C, Witwer KW, Aikawa E, Alcaraz MJ, Anderson JD, Andriantsitohaina $\mathrm{R}$, et al. Minimal information for studies of extracellular vesicles 2018 (MISEV2018): a position statement of the International Society for Extracellular Vesicles and update of the MISEV2014 guidelines. J Extracell Vesicles. 2018; 7(1):1535750

42. Konoshenko MY, Lekchnov EA, Vlassov AV, Laktionov PP. Isolation of Extracellular Vesicles: General Methodologies and Latest Trends. Biomed Res Int. 2018; 2018:8545347.

43. Thery C, Amigorena S, Raposo G, Clayton A. Isolation and characterization of exosomes from cell culture supernatants and biological fluids. Curr Protoc Cell Biol. 2006; Chapter 3:Unit 322.

44. Lasser C, Eldh M, Lotvall J. Isolation and characterization of RNA-containing exosomes. J Vis Exp. 2012; (59):e3037.

45. Witwer KW, Buzas EI, Bemis LT, Bora A, Lasser C, Lotvall $\mathrm{J}$, et al. Standardization of sample collection, isolation and analysis methods in extracellular vesicle research. J Extracell Vesicles. 2013; 2.

46. Zarovni N, Corrado A, Guazzi P, Zocco D, Lari E, Radano G, et al. Integrated isolation and quantitative analysis of exosome shuttled proteins and nucleic acids using immunocapture approaches. Methods. 2015; 87:46-58.

47. Alvarez ML, Khosroheidari M, Kanchi Ravi R, DiStefano JK. Comparison of protein, microRNA, and mRNA yields using different methods of urinary exosome isolation for the discovery of kidney disease biomarkers. Kidney Int. 2012; 82(9):1024-1032.

48. Taylor DD, Gercel-Taylor C. MicroRNA signatures of tumor-derived exosomes as diagnostic biomarkers of ovarian cancer. Gynecol Oncol. 2008; 110(1):13-21

49. Lane RE, Korbie D, Anderson W, Vaidyanathan R, Trau M. Analysis of exosome purification methods using a model liposome system and tunable-resistive pulse sensing. Sci Rep. 2015; 5:7639.

50. Freitas D, Balmana M, Pocas J, Campos D, Osorio H, Konstantinidi A, et al. Different isolation approaches lead to diverse glycosylated extracellular vesicle populations. J Extracell Vesicles. 2019; 8(1):1621131.

51. Soo CY, Song Y, Zheng Y, Campbell EC, Riches AC, Gunn-Moore F, et al. Nanoparticle tracking analysis monitors microvesicle and exosome secretion from immune cells. Immunology. 2012; 136(2):192-197.

52. Maroto R, Zhao Y, Jamaluddin M, Popov VL, Wang H, Kalubowilage M, et al. Effects of storage temperature on airway exosome integrity for diagnostic and functional analyses. J Extracell Vesicles. 2017; 6(1):1359478.

53. Mendt M, Kamerkar S, Sugimoto H, McAndrews KM, Wu CC, Gagea M, et al. Generation and testing of clinical-grade exosomes for pancreatic cancer. JCI Insight. 2018; 3(8).

54. Kalra H, Adda CG, Liem M, Ang CS, Mechler A, Simpson RJ, et al. Comparative proteomics evaluation of plasma exosome isolation techniques and assessment of the stability of exosomes in normal human blood plasma. Proteomics. 2013; 13(22):3354-3364.

55. Deanfield JE, Halcox JP, Rabelink TJ. Endothelial function and dysfunction: testing and clinical relevance. Circulation. 2007; 115(10):1285-1295.

56. Mudau M, Genis A, Lochner A, Strijdom H. Endothelial dysfunction: the early predictor of atherosclerosis. Cardiovasc J Afr. 2012; 23(4):222-231.

57. Wadley AJ, Veldhuijzen van Zanten JJ, Aldred S. The interactions of oxidative stress and inflammation with vascular dysfunction in ageing: the vascular health triad. Age (Dordr). 2013; 35(3):705-718.

58. Sun X, Feinberg MW. Regulation of endothelial cell metabolism: just go with the flow. Arterioscler Thromb Vasc Biol. 2015; 35(1):13-15. 
59. van Rooij E, Olson EN. MicroRNA therapeutics for cardiovascular disease: opportunities and obstacles. Nat Rev Drug Discov. 2012; 11(11):860-872.

60. Chen L, Wang Y, Pan Y, Zhang L, Shen C, Qin G, et al. Cardiac progenitor-derived exosomes protect ischemic myocardium from acute ischemia/reperfusion injury. Biochem Biophys Res Commun. 2013; 431(3):566-571.

61. Xiao J, Pan Y, Li XH, Yang XY, Feng YL, Tan HH, et al. Cardiac progenitor cell-derived exosomes prevent cardiomyocytes apoptosis through exosomal miR-21 by targeting PDCD4. Cell Death Dis. 2016; 7(6):e2277.

62. Ong SG, Lee WH, Huang M, Dey D, Kodo K, Sanchez-Freire V, et al. Cross talk of combined gene and cell therapy in ischemic heart disease: role of exosomal microRNA transfer. Circulation. 2014; 130(11 Suppl 1):S60-69.

63. Li J, Xue H, Li T, Chu X, Xin D, Xiong Y, et al. Exosomes derived from mesenchymal stem cells attenuate the progression of atherosclerosis in ApoE(-/-) mice via miR-let7 mediated infiltration and polarization of M2 macrophage. Biochem Biophys Res Commun. 2019; 510(4):565-572.

64. Kang K, Ma R, Cai W, Huang W, Paul C, Liang J, et al. Exosomes Secreted from CXCR4 Overexpressing Mesenchymal Stem Cells Promote Cardioprotection via Akt Signaling Pathway following Myocardial Infarction. Stem Cells Int. 2015; 2015:659890

65. Gonzalez-King H, Garcia NA, Ontoria-Oviedo I, Ciria M, Montero JA, Sepulveda P. Hypoxia Inducible Factor-1alpha Potentiates Jagged 1-Mediated Angiogenesis by Mesenchymal Stem Cell-Derived Exosomes. Stem Cells. 2017; 35(7):1747-1759.

66. Ibrahim AG, Cheng K, Marban E. Exosomes as critical agents of cardiac regeneration triggered by cell therapy. Stem Cell Reports. 2014; 2(5):606-619.

67. Vickers KC, Palmisano BT, Shoucri BM, Shamburek RD, Remaley AT. MicroRNAs are transported in plasma and delivered to recipient cells by high-density lipoproteins. Nat Cell Biol. 2011; 13(4):423-433.

68. Davidson SM, Riquelme JA, Zheng Y, Vicencio JM, Lavandero S, Yellon DM. Endothelial cells release cardioprotective exosomes that may contribute to ischaemic preconditioning. Sci Rep. 2018; 8(1):15885.

69. Zhan R, Leng X, Liu X, Wang X, Gong J, Yan L, et al. Heat shock protein 70 is secreted from endothelial cells by a non-classical pathway involving exosomes. Biochem Biophys Res Commun. 2009; 387(2):229-233.

70. Huang C, Han J, Wu Y, Li S, Wang Q, Lin W, et al. Exosomal MALAT1 derived from oxidized low-density lipoprotein-treated endothelial cells promotes M2 macrophage polarization. Mol Med Rep. 2018; 18(1):509-515.

71. Hergenreider E, Heydt S, Treguer K, Boettger T, Horrevoets AJ, Zeiher AM, et al. Atheroprotective communication between endothelial cells and smooth muscle cells through miRNAs. Nat Cell Biol. 2012; 14(3):249-256.

72. van Balkom BW, de Jong OG, Smits M, Brummelman J, den Ouden K, de Bree $\mathrm{PM}$, et al. Endothelial cells require miR-214 to secrete exosomes that suppress senescence and induce angiogenesis in human and mouse endothelial cells. Blood. 2013; 121(19):3997-4006, S3991-3915.

73. Njock MS, Cheng HS, Dang LT, Nazari-Jahantigh M, Lau AC, Boudreau E, et al. Endothelial cells suppress monocyte activation through secretion of extracellular vesicles containing antiinflammatory microRNAs. Blood. 2015; 125(20):3202-3212

74. Hou Z, Qin X, Hu Y, Zhang X, Li G, Wu J, et al. Longterm Exercise-Derived Exosomal miR-342-5p: A Novel Exerkine for Cardioprotection. Circ Res. 2019.

75. Zheng $B$, Yin WN, Suzuki $T$, Zhang $X H$, Zhang $Y$, Song LL, et al. Exosome-Mediated miR-155 Transfer from Smooth Muscle Cells to Endothelial Cells Induces Endothelial Injury and Promotes Atherosclerosis. Mol Ther. 2017; 25(6):1279-1294.

76. Ding Z, Wang X, Schnackenberg L, Khaidakov M, Liu S, Singla S, et al. Regulation of autophagy and apoptosis in response to ox-LDL in vascular smooth muscle cells, and the modulatory effects of the microRNA hsa-let-7 g. Int J Cardiol. 2013; 168(2):1378-1385.

77. Kapustin AN, Schoppet M, Schurgers LJ, Reynolds JL, McNair R, Heiss A, et al. Prothrombin Loading of Vascular Smooth Muscle Cell-Derived Exosomes Regulates Coagulation and Calcification. Arterioscler Thromb Vasc Biol. 2017; 37(3):e22-e32

78. Zhang C, Zhang K, Huang F, Feng W, Chen J, Zhang H, et al. Exosomes, the message transporters in vascular calcification. J Cell Mol Med. 2018; 22(9):4024-4033.

79. Rangrez AY, M'Baya-Moutoula E, Metzinger-Le Meuth V, Henaut L, Djelouat MS, Benchitrit J, et al. Inorganic phosphate accelerates the migration of vascular smooth muscle cells: evidence for the involvement of miR-223. PLoS One. 2012; 7(10):e47807.

80. Gui T, Zhou G, Sun Y, Shimokado A, Itoh S, Oikawa K, et al. MicroRNAs that target $\mathrm{Ca}(2+)$ transporters are involved in vascular smooth muscle cell calcification. Lab Invest. 2012; 92(9):1250-1259.

81. Kapustin AN, Chatrou ML, Drozdov I, Zheng Y, Davidson SM, Soong D, et al. Vascular smooth muscle cell calcification is mediated by regulated exosome secretion. Circ Res. 2015; 116(8):1312-1323.

82. Aikawa E. Extracellular vesicles in cardiovascular disease: focus on vascular calcification. J Physiol. 2016; 594(11):2877-2880

83. Wang $\mathrm{X}, \mathrm{Gu} \mathrm{H}$, Huang $\mathrm{W}$, Peng J, Li Y, Yang L, et al. Hsp20-Mediated Activation of Exosome Biogenesis in Cardiomyocytes Improves Cardiac Function and Angiogenesis in Diabetic Mice. Diabetes. 2016; 65(10):3111-3128.

84. Garcia NA, Moncayo-Arlandi J, Sepulveda P, Diez-Juan A. Cardiomyocyte exosomes regulate glycolytic flux in endothelium by direct transfer of GLUT transporters and glycolytic enzymes. Cardiovasc Res. 2016; 109(3):397-408.
85. Yang $Y$, Li Y, Chen X, Cheng X, Liao Y, Yu X. Exosomal transfer of miR-30a between cardiomyocytes regulates autophagy after hypoxia. J Mol Med (Berl). 2016; 94(6):711-724.

86. Wang X, Huang W, Liu G, Cai W, Millard RW, Wang Y, et al. Cardiomyocytes mediate anti-angiogenesis in type 2 diabetic rats through the exosomal transfer of miR-320 into endothelial cells. J Mol Cell Cardiol. 2014; 74:139-150.

87. $\mathrm{Hu} \mathrm{M}$, Guo $\mathrm{G}$, Huang $\mathrm{O}$, Cheng $\mathrm{C}, \mathrm{Xu} \mathrm{R}, \mathrm{Li} \mathrm{A}$, et al. The harsh microenvironment in infarcted heart accelerates transplanted bone marrow mesenchymal stem cells injury: the role of injured cardiomyocytes-derived exosomes. Cell Death Dis. 2018; 9(3):357.

88. Yu X, Deng L, Wang D, Li N, Chen X, Cheng X, et al. Mechanism of TNF-alpha autocrine effects in hypoxic cardiomyocytes: initiated by hypoxia inducible factor 1alpha, presented by exosomes. J Mol Cell Cardiol. 2012; 53(6):848-857.

89. Gao $\mathrm{W}$, Liu $\mathrm{H}$, Yuan J, Wu $\mathrm{C}$, Huang $\mathrm{D}, \mathrm{Ma} \mathrm{Y}$, et al. Exosomes derived from mature dendritic cells increase endothelial inflammation and atherosclerosis via membrane TNF-alpha mediated NF-kappaB pathway. J Cell Mol Med. 2016; 20(12):2318-2327.

90. Tang N, Sun B, Gupta A, Rempel H, Pulliam L. Monocyte exosomes induce adhesion molecules and cytokines via activation of NF-kappaB in endothelial cells. FASEB J. 2016; 30(9):3097-3106.

91. Li J, Zhang Y, Liu Y, Dai X, Li W, Cai X, et al. Microvesicle-mediated transfer of microRNA-150 from monocytes to endothelial cells promotes angiogenesis. J Biol Chem. 2013; 288(32):23586-23596.

92. De Silva N, Samblas M, Martinez JA, Milagro FI. Effects of exosomes from LPS-activated macrophages on adipocyte gene expression, differentiation, and insulin-dependent glucose uptake. J Physiol Biochem. 2018; 74(4):559-568.

93. Shimabukuro M, Hirata Y, Tabata M, Dagvasumberel M, Sato H, Kurobe H, et al. Epicardial adipose tissue volume and adipocytokine imbalance are strongly linked to human coronary atherosclerosis. Arterioscler Thromb Vasc Biol. 2013; 33(5):1077-1084.

94. Thomou T, Mori MA, Dreyfuss JM, Konishi M, Sakaguchi M, Wolfrum C, et al. Adipose-derived circulating miRNAs regulate gene expression in other tissues. Nature. 2017; 542(7642):450-455

95. Deng ZB, Poliakov A, Hardy RW, Clements R, Liu C, Liu Y, et al. Adipose tissue exosome-like vesicles mediate activation of macrophage-induced insulin resistance. Diabetes. 2009; 58(11):2498-2505

96. Wang F, Chen FF, Shang YY, Li Y, Wang ZH, Han L, et al. Insulin resistance adipocyte-derived exosomes aggravate atherosclerosis by increasing vasa vasorum angiogenesis in diabetic ApoE(-/-) mice. Int J Cardiol. 2018; 265:181-187.

97. Eguchi S, Takefuji M, Sakaguchi T, Ishihama S, Mori Y, Tsuda T, et al Cardiomyocytes capture stem cell-derived, anti-apoptotic microRNA-214 via clathrin-mediated endocytosis in acute myocardial infarction. J Biol Chem. 2019

98. Takafuji Y, Hori M, Mizuno T, Harada-Shiba M. Humoral factors secreted from adipose tissue-derived mesenchymal stem cells ameliorate atherosclerosis in Ldlr-/- mice. Cardiovasc Res. 2019; 115(6):1041-1051.

99. Caby MP, Lankar D, Vincendeau-Scherrer C, Raposo G, Bonnerot C. Exosomal-like vesicles are present in human blood plasma. Int Immunol. 2005; 17(7):879-887.

100. Srikanthan S, Li W, Silverstein RL, McIntyre TM. Exosome poly-ubiquitin inhibits platelet activation downregulates CD36 and inhibits pro-atherothombotic cellular functions. J Thromb Haemost. 2014; 12(11):1906-1917.

101. Li J, Tan M, Xiang Q, Zhou Z, Yan H. Thrombin-activated platelet-derived exosomes regulate endothelial cell expression of ICAM-1 via microRNA-223 during the thrombosis-inflammation response. Thromb Res. 2017; 154:96-105.

102. Tan M, Yan HB, Li JN, Li WK, Fu YY, Chen W, et al. Thrombin Stimulated Platelet-Derived Exosomes Inhibit Platelet-Derived Growth Factor Receptor-Beta Expression in Vascular Smooth Muscle Cells. Cell Physiol Biochem. 2016; 38(6):2348-2365

103. Vicencio JM, Yellon DM, Sivaraman V, Das D, Boi-Doku C, Arjun S, et al. Plasma exosomes protect the myocardium from ischemia-reperfusion injury. J Am Coll Cardiol. 2015; 65(15):1525-1536.

104. Giricz Z, Varga ZV, Baranyai T, Sipos P, Paloczi K, Kittel A, et al Cardioprotection by remote ischemic preconditioning of the rat heart is mediated by extracellular vesicles. J Mol Cell Cardiol. 2014; 68:75-78.

105. Yamaguchi T, Izumi Y, Nakamura Y, Yamazaki T, Shiota M, Sano S, et al. Repeated remote ischemic conditioning attenuates left ventricular remodeling via exosome-mediated intercellular communication on chronic heart failure after myocardial infarction. Int J Cardiol. 2015; 178:239-246.

106. Li J, Rohailla S, Gelber N, Rutka J, Sabah N, Gladstone RA, et al. MicroRNA-144 is a circulating effector of remote ischemic preconditioning. Basic Res Cardiol. 2014; 109(5):423.

107. Kuwabara Y, Ono K, Horie T, Nishi H, Nagao K, Kinoshita M, et al. Increased microRNA-1 and microRNA-133a levels in serum of patients with cardiovascular disease indicate myocardial damage. Circ Cardiovasc Genet. 2011; 4(4):446-454

108. Cheng Y, Wang X, Yang J, Duan X, Yao Y, Shi X, et al. A translational study of urine miRNAs in acute myocardial infarction. J Mol Cell Cardiol. 2012; 53(5):668-676.

109. Matsumoto S, Sakata Y, Suna S, Nakatani D, Usami M, Hara M, et al Circulating p53-responsive microRNAs are predictive indicators of heart failure after acute myocardial infarction. Circ Res. 2013; 113(3):322-326. 
110. Gao L, Gregorich ZR, Zhu W, Mattapally S, Oduk Y, Lou X, et al. Large Cardiac Muscle Patches Engineered From Human Induced-Pluripotent Stem Cell-Derived Cardiac Cells Improve Recovery From Myocardial Infarction in Swine. Circulation. 2018; 137(16):1712-1730.

111. Gallet R, Dawkins J, Valle J, Simsolo E, de Couto G, Middleton R, et al. Exosomes secreted by cardiosphere-derived cells reduce scarring, attenuate adverse remodelling, and improve function in acute and chronic porcine myocardial infarction. Eur Heart J. 2017; 38(3):201-211.

112. de Couto G, Gallet R, Cambier L, Jaghatspanyan E, Makkar N, Dawkins JF, et al. Exosomal MicroRNA Transfer Into Macrophages Mediates Cellular Postconditioning. Circulation. 2017; 136(2):200-214.

113. Cheng M, Yang J, Zhao X, Zhang E, Zeng Q, Yu Y, et al. Circulating myocardial microRNAs from infarcted hearts are carried in exosomes and mobilise bone marrow progenitor cells. Nat Commun. 2019; 10(1):959.

114. Bi S, Wang C, Jin Y, Lv Z, Xing X, Lu Q. Correlation between serum exosome derived miR-208a and acute coronary syndrome. Int J Clin Exp Med. 2015; 8(3):4275-4280.

115. Emanueli C, Shearn AI, Laftah A, Fiorentino F, Reeves BC, Beltrami C, et al. Coronary Artery-Bypass-Graft Surgery Increases the Plasma Concentration of Exosomes Carrying a Cargo of Cardiac MicroRNAs: An Example of Exosome Trafficking Out of the Human Heart with Potential for Cardiac Biomarker Discovery. PLoS One. 2016; 11(4):e0154274. 